\title{
Aggressive Maneuvering Flight Tests of a Miniature Robotic Helicopter
}

\author{
Vladislav Gavrilets, Ioannis Martinos, Bernard Mettler, and Eric Feron \\ Massachusetts Institute of Technology, Cambridge MA 02139, USA
}

\begin{abstract}
In this paper we describe the control logic that enabled a small-scale unmanned helicopter to execute an aerobatic maneuver under computer control. The logic consists of steady-state trim trajectory controllers, used prior to, and upon the exit from the maneuver; and a maneuver logic inspired by human pilot strategies for maneuver execution. Extensive flight tests with this control logic demonstrated smooth entry into the maneuver, automatic recovery from the maneuver to a steady-state trim trajectory, and the robustness of the trim-trajectory control system toward measurement and modelling errors. This approach can be used with an advanced motion planning algorithm which utilizes the vehicle agility.
\end{abstract}

\section{Introduction}

Miniature helicopters with a stiff hub allow expert R/C pilots to perform aggressive maneuvers, like axial rolls, hammerheads, and split-S. The agility can be useful for an unmanned surveillance helicopter in a confined environment, such as a mountain valley or a city. To control an agile vehicle from a remote location, a high level of autonomy has to be incorporated into the flight control system. Until now, aerobatic capability has not been achieved in autonomous flight.

Our group at MIT has demonstrated an autonomous axial roll with an X-Cell 60 helicopter [7]. This work follows our previous effort in studying human pilot's execution of the maneuvers [2]. We have analyzed time histories of pilot commands and state variables during several maneuvers, devised human-inspired maneuver execution strategies, and showed in a hardware-inthe-loop simulation how some of those maneuvers can be performed automatically. The automatic axial roll first performed in November 2001 provided an experimental validation of the control algorithms; the implementation details and flight tests results are presented in this paper. In an earlier paper [3] we presented the development and validation of the nonlinear dynamic model that adequately describes the helicopter in aerobatic flight; the model played a key role in the successful implementation of an autonomous aerobatic maneuver.

The helicopter features a $5 \mathrm{ft}$ diameter hingeless main rotor with a BellHiller stabilizer bar. Total weight of the helicopter including the $7 \mathrm{lb}$ avionics box is $17 \mathrm{lbs}$. The helicopter is equipped with an electronic governor, which maintains the rotor speed at $1600 \mathrm{rpm}$. The sensor package [7] includes three gyros, three accelerometers, a GPS receiver, an altimeter, and a compass. 
Table 1 shows modes of operation in our current flight control system. In the "manual" mode the pilot commands the collective and cyclic pitch deflections, as well as a setpoint for a yaw rate feedback controller. In the "rate tracking" mode the pilot commands all three angular rates and the collective pitch. In the "velocity/heading rate command - altitude hold" (VHRCAH) mode the pilot commands turn rate, forward, side and vertical velocities or engages altitude hold. In the "hover hold" mode the helicopter maintains GPS position, pressure altitude, and magnetic heading. Finally, the pilot can initiate an automatic axial roll maneuver by activating a transmitter switch.

Table 1. Autopilot Modes for MIT X-Cell Helicopter

\begin{tabular}{l|llll}
\hline & Manual & Rate Tracking & VHRCAH & Hover hold \\
\hline Longitudinal & Lon. Cyclic & Pitch rate & Forward velocity & Position \\
Lateral & Lat. Cyclic & Roll rate & Side velocity & Position \\
Directional & Yaw rate & Yaw rate & Turn rate & Heading \\
Vertical & Collective & Collective & Altitude/rate & Altitude \\
\hline
\end{tabular}

In this paper we will first describe the longitudinal-vertical and lateraldirectional control systems used for the VHRCAH mode. Next we describe the human-inspired control logic [2], which enabled the automatic axial roll maneuver. The paper concludes by outlining how these control laws will be used in a guidance system designed to exploit the natural agility of such vehicles [1].

\section{Longitudinal-vertical controller}

The command variables are body-axis forward and inertial vertical velocities. The pilot selects forward speed setting by fore-aft deflections of the left stick on the remote control. Fore-aft motion of the spring-loaded right stick is used for altitude rate command, or for engaging altitude hold.

\subsection{Linearized longitudinal-vertical dynamics}

Longitudinal and lateral dynamics of the X-Cell are sufficiently decoupled [3] to design separate feedback controllers. A linear model of the longitudinalvertical dynamics was obtained by an analytical linearization of the nonlinear equations of motion around a steady turning flight at a constant altitude.

The state vector of perturbations from the trim conditions is $X_{\text {lon }}=$ $\left[\begin{array}{llll}u & a_{1} & w & q\end{array}\right]^{T}$, where $u$ is the perturbation of the body axis forward speed from the trim speed $U_{e}, a_{1}$ is the longitudinal flapping angle of the main rotor, 
$w$ is the body Z-axis velocity, $q$ is the pitch rate, and $\theta$ is the pitch angle. The vector of control input perturbations is $U_{l o n}=\left[\begin{array}{ll}\delta_{l o n} & \delta_{c o l}\end{array}\right]^{T}$, where $\delta_{l o n}$ is the longidutinal cyclic pitch, and $\delta_{c o l}$ is the collective pitch of the main rotor blades. The equations of motion are given in Eq. 1:

$$
\begin{aligned}
\dot{u}= & X_{u}\left(u-u_{w}\right)-\frac{g}{\cos \Phi_{e}} a_{1}-U_{e} \tan \Theta_{e} q-g \cos \Theta_{e} \theta \\
\dot{a_{1}}= & \frac{1}{\tau_{e}} \frac{\partial a_{1}}{\partial \mu} \frac{u-u_{w}}{\Omega R}-\frac{a_{1}}{\tau_{e}}+\frac{1}{\tau_{e}} \frac{\partial a_{1}}{\partial \mu_{z}} \frac{w-w_{w}}{\Omega R}-q+\frac{K_{\delta_{l o n}}}{\tau_{e}} \delta_{l o n}+\frac{1}{\tau_{e}} \frac{\partial a_{1}}{\partial \delta_{c o l}} \delta_{c o l} \\
\dot{w}= & Z_{u}\left(u-u_{w}\right)+Z_{w}\left(w-w_{w}\right)+\left(Z_{q}^{h_{t}}+U_{e}\right) q-g \cos \Phi_{e} \sin \Theta_{e} \theta \\
& +Z_{c o l} \delta_{c o l} \\
\dot{q}= & M_{a_{1}} a_{1}+M_{w}^{h_{t}}\left(w-w_{w}\right)+M_{q}^{h_{t}} q, \dot{\theta}=\cos \Phi_{e} q
\end{aligned}
$$

Here $\Phi_{e}$ is the trim value of the bank angle; $\Theta_{e}-$ the trim pitch angle, $\Omega R$ is the main rotor blade tip speed, $u_{w}$ and $w_{w}$ are wind velocities. Aerodynamic coefficients are obtained by linearization of the nonlinear model near the selected equilibrium point, e.g. $X_{u}=\frac{1}{m} \frac{\partial X\left(U_{e}, \Phi_{e}\right)}{\partial U} \cdot \tau_{e}$ is the effective time constant of the rotor [3].

\subsection{Model reduction for control system design}

To make use of the robustness properties of the linear quadratic regulator, a measurement of the full state vector is needed. When this is impossible, an estimator with a significantly higher bandwidth than the open-loop plant dynamics is known to provide good robustness properties in practice. Forward velocity, ascent rate, pitch angle and pitch rate can be either measured or estimated in real-time. Rotor flapping angles, on the other hand, are difficult to measure and their estimation would rely on an accurate knowledge of the open-loop dynamics, leading to the loss of controller robustness. Instead, we decided to use a rigid-body approximation of the longitudinal-vertical dynamics. The flapping state participates in a lightly damped fuselage-rotor mode [5]. To eliminate potential gain margin problems with the controller design based on the reduced-order rigid-body model, we used a notch filter on the longitudinal cyclic [4]. The reduced $4^{t h}$ order dynamics should approximate the plant with the notch filter on the longitudinal cyclic. The equations of motion for the reduced order model are

$$
\begin{aligned}
\dot{u}= & X_{u}\left(u-u_{w}\right)+X_{w}^{m r}\left(w-w_{w}\right)-g \cos \Theta_{e} \theta+\left(X_{q}^{m r}-U_{e} \tan \Theta_{e}\right) q \\
& +X_{\delta_{l o n}}^{m r} \delta_{l o n}+X_{c o l}^{m r} \delta_{c o l} \\
\dot{w}= & Z_{u}\left(u-u_{w}\right)+Z_{w}\left(w-w_{w}\right)+\left(Z_{q}^{h_{t}}+U_{e}\right) q-g \cos \Phi_{e} \sin \Theta_{e} \theta \\
& +Z_{\delta_{l o n}} \delta_{l o n}+Z_{c o l} \delta_{c o l} \\
\dot{q}= & M_{u}^{m r}\left(u-u_{w}\right)+\left(M_{w}^{m r}+M_{w}^{h_{t}}\right)\left(w-w_{w}\right)+\left(M_{q}^{m r}+M_{q}^{h_{t}}\right) q \\
& +M_{\delta_{l o n}}^{m r} \delta_{l o n}+M_{c o l}^{m r} \delta_{c o l}, \dot{\theta}=\cos \Phi_{e} q
\end{aligned}
$$


The rate damping derivative $M_{q}^{m r}$ was chosen to match the frequency at which the notched plant attains 45 degree phase lag [4]. Other stability and control derivatives can be calculated by using a static relationship instead of the differential equation for flapping in Eq. 1.

\subsection{Control system design}

To achieve zero steady-state error in forward speed and altitude rate tracking the reduced state vector was augmented with the integrators, which resulted in a new 6 -dimensional state vector. The linearized equations for the forward and vertical speed integral errors are:

$$
\begin{aligned}
\dot{u}_{i} & =u^{c m d}-u \\
\dot{V}_{h i} & =V_{h}^{c m d}-u \sin \Theta_{e}+w \cos \Theta_{e} \cos \Phi_{e}-\theta U_{e}\left(\cos \Theta_{e}+\frac{\sin ^{2} \Theta_{e} \cos \Phi_{e}}{\cos \Theta_{e}}\right)
\end{aligned}
$$

The feedback gains for the augmented system were calculated with a linear quadratic regulator approach. Diagonal $Q$ and $R$ matrices were used in the quadratic cost function:

$$
J=\int_{0}^{\infty}\left(x^{T} Q x+u^{T} R u\right) d t
$$

The controllers were designed for the following six trim points: $0,3,6,9,12$ and $15 \mathrm{~m} / \mathrm{sec}$ body axis forward speed. All design cases were based on a trim bank angle of 45 degrees. Based on simulation studies with the full model we eliminated several feedback gains. Feedforward signals were added to improve transient response. Figure 1 shows the resulting control architecture.

The control laws were designed to the following specifications: the damping ratio of at least 0.5 for the two oscillatory modes with the frequencies of less than $10 \mathrm{rad} / \mathrm{sec}$; rise time for the altitude-rate step response of no more than 1 second; rise time for the forward-speed step response of no more than 2.5 seconds. The requirement for the constant-gain proportional altitude hold controller was maximum \pm 3 meter error during 45 degree banks.

\subsection{Flight test results for longitudinal-vertical controller}

Flight testing showed that the longitudinal-vertical closed loop dynamics was predicted well by the simulation. Figure 2 shows the forward velocity and altitude time histories during rapid acceleration and deceleration maneuvers.

The forward velocity estimate is subject to an average of $1 \mathrm{~m} / \mathrm{sec}$ jumps. They are due to the GPS corrections, which arrive once a second with one second latency [7]. During this flight, winds up to 10 knots were recorded. The control system proved to be robust to such measurement errors and flight conditions. 


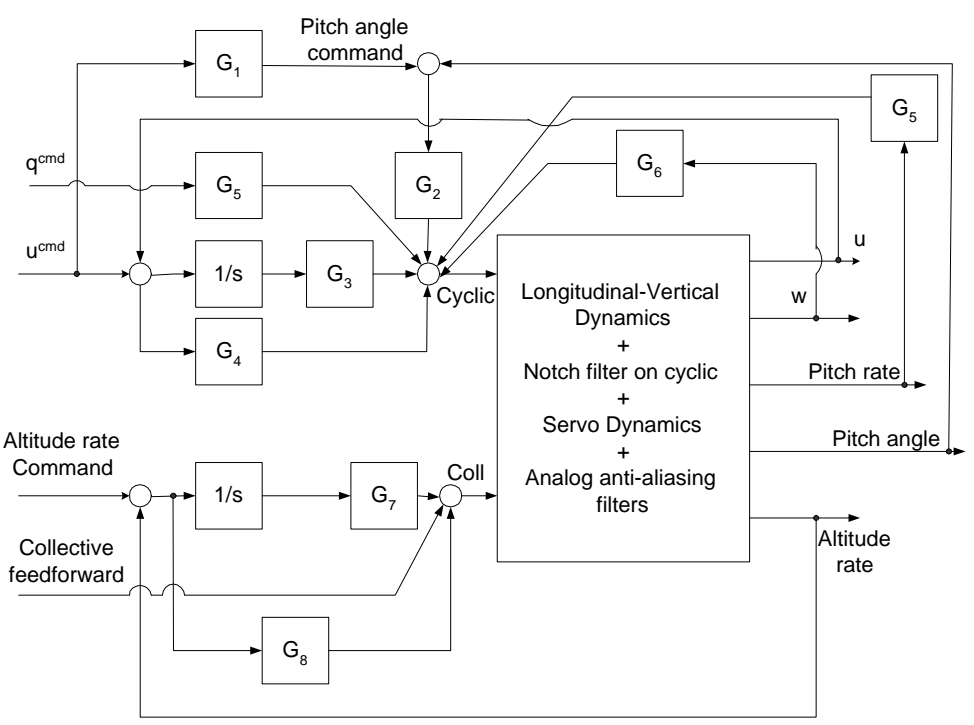

Fig. 1. Longitudinal-vertical control architecture
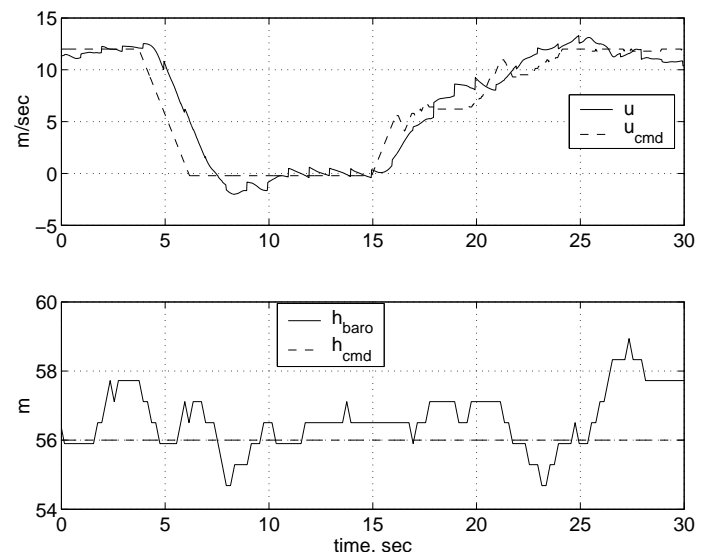

Fig. 2. Forward velocity and altitude time histories during rapid acceleration and deceleration

\section{Lateral-directional controller}

The lateral controller was described in detail in our previous publication [4]; here we provide a summary of its main features. A block-diagram of the controller is shown in Figure 3. 


\subsection{Command variables}

We chose the yaw rate and the sideslip velocity as the command variables. The yaw rate command is mechanized as a turn rate command: it acts as the yaw rate command at hover and as an effective bank angle command in forward flight. The control logic uses the tail rotor to turn at hover, and primarily the lateral cyclic in forward flight, utilizing the tail rotor for the turn coordination. The pilot commands turn rate by a sideways deflection of the right stick, and side velocity by a sideways motion of the left stick. Both sticks are spring-loaded.

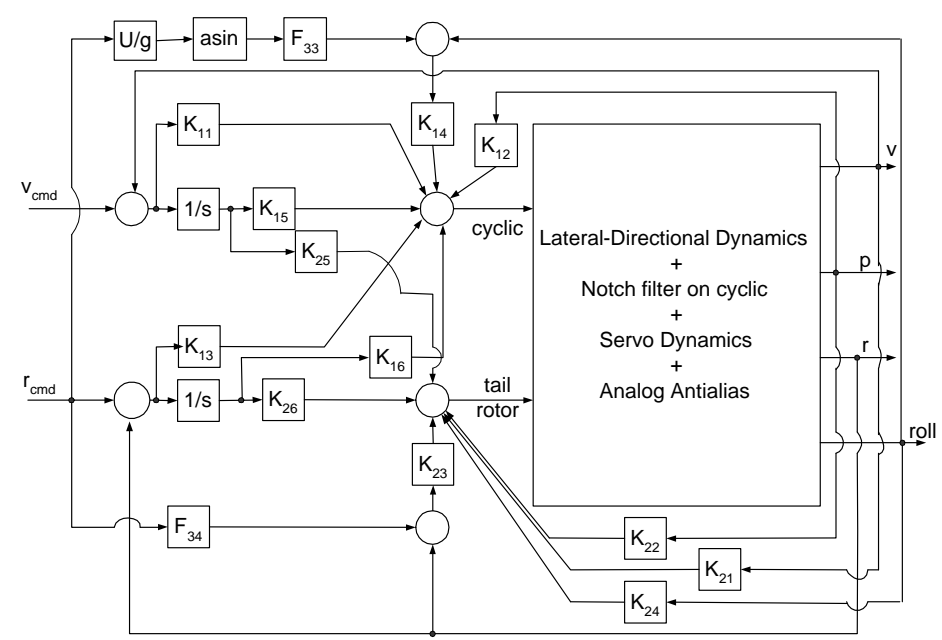

Fig. 3. Lateral-directional controller structure

\subsection{Linear model and control design}

X-Cell's lateral-directional dynamics are adequately described by a linear model with five states and two inputs. The state vector is $x=\left[\begin{array}{ll}v b & p\end{array}\right]^{T}$, where $v$ is the side velocity, $b$ is the lateral rotor flapping, $p$ is the roll rate, $r$ is the yaw rate, and $\phi$ is the roll angle. The control inputs are the lateral cyclic $\delta_{a}$ and the tail rotor pitch $\delta_{r}$. The main rotor torque variation due to collective pitch deflection $\delta_{c o l}$ is treated as a disturbance. Another source of disturbance is wind side velocity $v_{w}$. The linearized equations of motion for straight and level flight are given in Eq. 3

$$
\begin{aligned}
\dot{v} & =Y_{v}\left(v-v_{w}\right)+g \cdot b+W_{e} p+\left(Y_{r}-U_{e}\right) r+g \cos \left(\Theta_{e}\right) \phi+Y_{\delta_{r}} \delta_{r} \\
\dot{b} & =-\frac{1}{\tau_{e}} b-p-B_{\mu} \frac{v-v_{w}}{\Omega R_{m r}}+\frac{B_{l a t}}{\tau_{e}} \delta_{a}
\end{aligned}
$$




$$
\begin{aligned}
& \dot{p}=L_{b} b+L_{v}^{e}\left(v-v_{w}\right)+L_{r} r+L_{\delta_{r}} \delta_{r} \\
& \dot{r}=N_{v}\left(v-v_{w}\right)+N_{p} p+N_{r} r+N_{\delta_{r}} \delta_{r}+N_{\delta_{c o l}} \delta_{c o l} \\
& \dot{\phi}=p+\tan \left(\Theta_{e}\right) r
\end{aligned}
$$

Similarly to the longitudinal case, a notch filter on the lateral cyclic was applied to retain gain margin at the natural frequency of the lightly damped fuselage-rotor mode [4]. The notched plant was approximated with the rigid body model, and the integrators were appended on the command variable tracking errors. Linear-quadratic regulator gains were computed for the resulting $6 \times 2$ system for six forward speed values from 0 to $15 \mathrm{~m} / \mathrm{sec}$. Controller gains were switched once a new design point velocity was reached; hysteresis logic was used to prevent limit-cycling. Continuity of control surface commands during gain switching (as well as during switching between different controller modes) was ensured by resetting the integrator states to appropriate values. This is possible when the control system has at least as many integrators as control surface inputs. This gain scheduling logic proved superior to a linear gain interpolation in transition between hover and forward flight, when lateral-directional gains change rapidly with speed.

The turn rate command was limited to preclude a commanded bank angle above 45 degrees. A feedforward logic was used to assure a crisp bank angle response in forward flight, and a fast yaw rate response in hovering flight [4].

The control laws were tested from hover to $15 \mathrm{~m} / \mathrm{sec}$ forward flight. The closed loop response was adequately predicted by the simulation [4].

\section{Maneuver execution logic}

The logic for automatic execution of an axial roll was inspired by the human pilot's strategy for executing the maneuver [2]. Based on input and state time histories recorded during a series of manual aerobatic maneuvers we made the following observation: maneuvers are repeatable; the pilot uses few continuous feedbacks during the maneuvers; most of the pilot's actions can be described by piece-wise constant or piece-wise linear functions, with the switching times based on vehicle attitude.

The pilot's strategy for implementing the axial roll can be described as follows [2]. Prior to the maneuver the pilot slightly pitches up the helicopter to make use of the translational lift while inverted. Next, the pilot ramps up the lateral cyclic to full deflection and modulates the collective roughly as a cosine of the bank angle; this keeps the rotor lift force positive when the helicopter is upside down, and generates little thrust when the rotor disk is normal to the horizon. When the helicopter reaches a specific attitude $(\approx-50$ degrees), the pilot brings the cyclic to neutral.

We decided to use three decoupled proportional-integral controllers to track angular rate trajectories; notch filters on cyclic commands were used to attenuate the lightly damped fuselage-rotor mode [5]. The pilot performed 
a number of axial rolls with the rate tracking loops, based on which we generated reference trajectories for the rates and the collective pitch angle. The pitch and yaw rate commands were zero throughout the maneuver. The roll rate trajectory approximated an actual trajectory during manual axial rolls, with 0.2 seconds coasting time to arrest the roll motion after the maneuver. The collective angle was modulated as a cosine of the bank angle, with a limit on negative value of collective to retain large control authority in the inverted condition.

The axial roll maneuvers under computer control were performed in the following way. The pilot took off manually, and engaged the "velocity/heading rate command/altitude hold" mode at a safe maneuver altitude (above 50 $\mathrm{m})$. Then he commanded the forward speed setpoint for maneuver execution (12 and $15 \mathrm{~m} / \mathrm{sec}$ were used). From that point the helicopter was flown with a single left sideways stick (turn rate command), while the autopilot maintained the altitude and forward speed setpoints. The pilot brought the helicopter to level flight by commanding zero turn rate, and engaged an automatic axial roll sequence. The helicopter performed the roll, and exited the maneuver sequence at a level attitude and close to zero roll rate. At this point the controller automatically went back to the "velocity/heading rate command/altitude hold" mode. The altitude hold acquired a new altitude setpoint, and the forward speed returned to the speed setting prior to the maneuver entry. A number of rolls were performed. All state trajectories were similar and matched those predicted by the simulation. Maximum altitude loss of about 20 meters along with a forward speed increase to $20 \mathrm{~m} / \mathrm{sec}$ occurred during axial rolls performed at $15 \mathrm{~m} / \mathrm{sec}$. A smaller altitude drop was observed with the commanded entry speed of $12 \mathrm{~m} / \mathrm{sec}$. These effects were also captured by the simulation. Heading change was small in all maneuvers.

Smooth switching between controller modes is enabled by proper initialization of integrator states as described in Section 3.2.

Figure 4 shows the state trajectories during an autonomous axial roll. We can see that most of the altitude loss and gain in speed occurs during the second half of the roll. We did not use the pitch up before the maneuver, which contributed to the loss of altitude. A forward speed tracking controller can be used during the roll to prevent speed up and limit loss of altitude. Figure 5 shows the altitude and speed time histories for a longer time interval to illustrate the recovery after the maneuver completion.

\section{Guidance System For Agile Maneuvering}

To take advantage of the inherent agility of the vehicle, a guidance system which accounts for the full vehicle performance is required. The computation of an optimal (e.g. fastest) trajectory between two points based on complete vehicle dynamics is in general computationally intractable because of the high order of helicopter equations of motion. Frazzoli et al. [1] introduced 


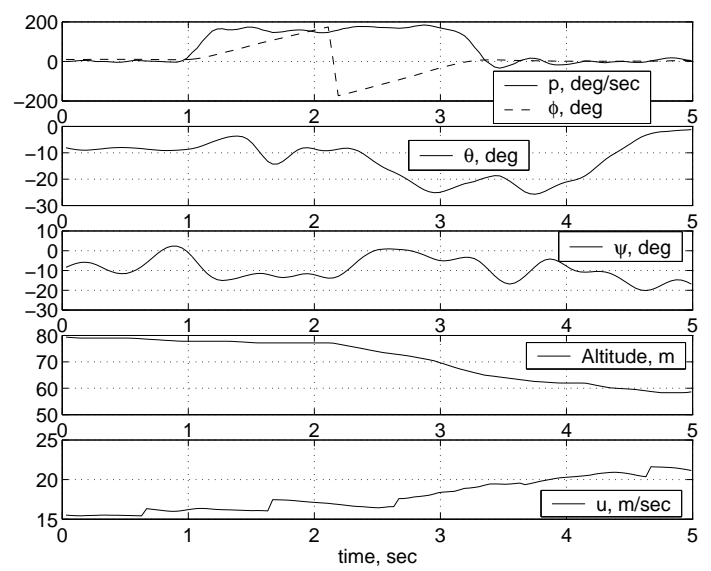

Fig. 4. State trajectory during autonomous axial roll
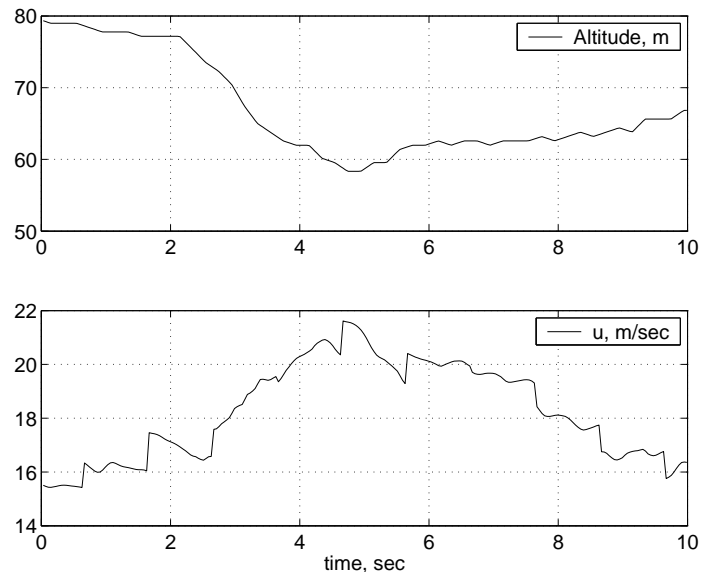

Fig. 5. Altitude and speed during the axial roll and recovery phase

a motion planning technique based on a maneuver automaton, in which the vehicle motion is represented by trim trajectories and maneuvers. The trim trajectories correspond to the states of the automaton, and the maneuvers to finite duration transitions between the trim states. The motion planning then amounts to finding a sequence of trim trajectories and maneuvers, which minimizes the chosen performance criteria. A sequential decision problem is solved with dynamic programming. A value iteration is used to compute the cost-to-go function. This function, which can be computed offline and used to generate trajectories in real-time, gives the cost (e.g. travel time) as a function of the initial vehicle state and a goal state. The details of building a maneuver automaton and cost function computation for the X-Cell helicopter are described in [6]. 
Such a guidance algorithm requires precisely the characteristics of the control logic presented in this paper: a tight trim trajectory tracking, accurate maneuver execution, and guaranteed recovery from the maneuvers.

\section{Conclusions}

An aerobatic maneuver under computer control was demonstrated with a miniature helicopter. The control laws for performing an autonomous axial roll were presented. Similar control laws for more advanced maneuvers are being developed and will be flight tested.

\section{Acknowledgements}

We would like to thank MIT graduate students Kara Sprague and Alex Shterenberg, and Dr. David Vos of Athena Technologies for their contributions to the project. Partial funding for this research was provided by the Office of Naval Research under Young Investigator Award and the NASA Grant NAG2-1441.

\section{References}

1. E. Frazzoli, M.Dahleh, and E. Feron. Real-time motion planning for agile autonomous vehicles. AIAA Journal of Guidance, Control and Dynamics, 25(1):116-129, 2002.

2. V. Gavrilets, E. Frazzoli, B. Mettler, M. Piedmonte, and E. Feron. Aggressive maneuvering of small autonomous helicopters: A human-centered approach. International Journal of Robotics Research, pages 795-807, October 2001.

3. V. Gavrilets, B. Mettler, and E. Feron. Nonlinear model for a small-size acrobatic helicopter. Number AIAA 2001-4333, Montreal, Canada, August 2001. Proceedings of the AIAA Guidance, Navigation, and Control Conference.

4. B. Mettler, V. Gavrilets, E. Feron, and T. Kanade. Dynamic compensation for high-bandwidth control of small-scale helicopter. San Francisco, CA, January 2002. American Helicopter Society Specialist Meeting.

5. B. Mettler, M.B. Tischler, and T. Kanade. System identification modeling of a small-scale unmanned rotorcraft for control design. Journal of the American Helicopter Society, 47(1):50-63, January 2002.

6. B. Mettler, M. Valenti, T. Schouwenaars, E. Frazzoli, and E. Feron. Rotorcraft motion planning for agile maneuvering. Montreal, Canada, June 2002. Proceedings of the 55th Forum of the American Helicopter Society.

7. K. Sprague, V. Gavrilets, D. Dugail, B. Mettler, and E. Feron. Design and applications of an avionics system for a miniature acrobatic helicopter. Daytona Beach, FL, 2001. AIAA Digital Avionics Systems Conference. 